\title{
GLOBAL EXISTENCE AND SINGULARITY FORMATION IN SOLUTIONS OF A MODIFIED FOURIER LAW
}

\author{
BY \\ K. SAXTON \\ Loyola University, New Orleans, Louisiana
}

\begin{abstract}
The aim of this paper is the analysis of formation of singularities from smooth initial data, for rigid conductors. The modified Fourier law used asserts that the governing second-order equation is hyperbolic with first-order dissipation. Global existence for small initial data is also examined.
\end{abstract}

1. Introduction. The model for heat conduction used here replaces the classical Fourier law with a modification introduced by Kosinski [4], physically motivated by Cimmelli and Kosinski [1]. In this approach the heat flux is proportional to the gradient of a "new" temperature scale $\beta$. This temperature $\beta$ is related to the absolute temperature $\vartheta>0$ by the initial-value problem

$$
\begin{aligned}
\beta_{t} & =f(\vartheta, \beta), \\
\beta(0) & =\beta_{0}, \quad \beta \in(0, \infty) .
\end{aligned}
$$

The heat flux $\mathbf{q}$ is related to $\nabla \beta$ by

$$
\mathbf{q}=d \nabla \beta
$$

Generally $d$, for rigid conductors, may be a function of $\vartheta$.

We employ the following constitutive equations for nonlinear heat conductors $([4])$ :

$$
\psi=\hat{\psi}(\vartheta, \nabla \beta)
$$

and

$$
\eta=-\partial_{\vartheta} \hat{\psi}(\vartheta, \nabla \beta)
$$

where $\psi$ is the Helmholtz free energy and $\eta$ the specific entropy. For an explicit function $f$ (cf. [4]) in Eq. (1.1), the Maxwell-Cattaneo relation can be obtained: $\tau \mathbf{q}_{t}+\mathbf{q}=-k \nabla \vartheta$,

Received June 22, 1994.

1991 Mathematics Subject Classification. Primary 35L10, 35L67, 73B30.

Partially supported by the LEQSF Grant (1991-94)-RD-A-22 
with $\tau$ the relaxation time depending on $\vartheta$ and $\beta$ (cf. [4]). We will assume that the free energy $\hat{\psi}$ is given by

$$
\hat{\psi}(\vartheta, \nabla \beta)=\hat{\psi}_{1}(\vartheta)+\frac{1}{2} \hat{\varepsilon}_{2}(\nabla \beta)^{2}
$$

where $\hat{\varepsilon}_{2}=k_{0} \tau_{0} /\left(\rho_{0} \vartheta^{0}\right)$, and $\tau_{0}, k_{0}$ are characteristic material constants representing the relaxation time and thermal conductivity at constant reference temperature $\vartheta^{0}, \beta^{0}$ and $\rho_{0}$ is a mass density. Additionally, we assume that the function in Eq. (1.2) is a constant $d=k_{0}$.

From the energy balance law, one obtains a second-order hyperbolic equation. In the case of a one-dimensional rigid body (cf. Kosinski and Saxton [6]) this becomes

$$
-C\left(\beta, \beta_{t}\right) \beta_{t t}+b \beta_{x} \beta_{x t}+a \beta_{x x}+H\left(\beta, \beta_{t}\right)=0
$$

where

$$
\begin{aligned}
C\left(\beta, \beta_{t}\right) & =\rho_{0} \tau_{0} \vartheta c_{V}(\vartheta) \\
H\left(\beta, \beta_{t}\right) & =\rho_{0} \vartheta c_{V}(\vartheta) f_{0}^{\prime}(\beta) \beta_{t} \\
a & =k_{0} \vartheta^{0} \\
b & =\tau_{0} k_{0}
\end{aligned}
$$

and

$$
\begin{aligned}
\tau_{0} f(\vartheta, \beta) & =\vartheta^{0} \log \left(\vartheta^{0} / \vartheta\right)+f_{0}(\beta) \\
f_{0}(\beta) & =\tau_{0} f\left(\vartheta^{0}, \beta\right)
\end{aligned}
$$

Here $c_{V}=\vartheta \partial_{\vartheta} \eta>0$ is the specific heat at constant volume, and the relations (1.7) are derived by Kosinski in [4]. Equation (1.5) can be rewritten as a system of hyperbolic equations by introducing new dependent variables

$$
\begin{aligned}
& \beta_{t}=w \\
& \beta_{x}=p
\end{aligned}
$$

Then

$$
\begin{gathered}
-C(\beta, w) w_{t}+b p w_{x}+a p_{x}+H(\beta, w)=0, \\
\beta_{t}-w=0, \\
p_{t}-w_{x}=0 .
\end{gathered}
$$

Kosinski and Saxton [6] showed that the amplitude $\alpha=\left[w_{t}\right]$ of acceleration waves satisfies a Bernoulli-type equation

$$
\frac{d}{d t} \alpha-n \tau_{0} \alpha^{2}+\frac{1}{m \tau_{0}} \alpha=0 .
$$

Here $n, m$ are constants depending on the value of $\vartheta$ and $\beta$ in front of the wave, assumed to be a constant state. Both $n$ and $m$ are positive. As a result, the solution $\alpha(t)$ of Eq. (1.10) blows up to $+\infty$ or $-\infty$ in finite time provided that the initial condition $\alpha_{0}=\alpha(0)$ satisfies the inequality

$$
-n \tau_{0} \alpha_{0}+\frac{1}{m \tau_{0}}<0
$$


Here the initial jump in $w_{t}$ and $w_{x}$ results in $\left[w_{t}\right]$ and $\left[w_{x}\right]$ becoming unbounded in finite time. Similar results were obtained in [6] for elastic conductors where both amplitudes $\left[w_{t}\right]$ and $\left[v_{t}\right]$ ( $v$ is a velocity) also satisfy a Bernoulli-type equation.

In Sec. 2 we give an existence result based on Nishida [12] for global in time, small amplitude solutions. Section 3 then examines the breakdown of solutions from smooth initial data in the sense that $(w, p)$ are no longer of class $C^{1}(\mathbb{R})^{2}$ after some time $t^{*}<\infty$.

2. Global smooth solution. In this section we obtain sufficient conditions for global existence of small amplitude solutions of the Cauchy problem for Eq. (1.5).

We assume that the function $f_{0}$ in Eqs. (1.7) is linear, that is,

$$
f_{0}(\beta)=-\left(\beta-\beta^{0}\right)
$$

A local existence theorem was established by Cimmelli and Kosinski [2]. The initial conditions for the heat propagation (1.5) can be given physically for $\beta$ and $\vartheta$. Using the evolution equation (1.1), and (1.7) with (2.1), these can be obtained as:

$$
\begin{aligned}
\beta(0, x) & =\beta_{0}(x) \\
\beta_{t}(0, x) & =\frac{\vartheta^{0}}{\tau_{0}} \log \frac{\vartheta^{0}}{\vartheta(0, x)}-\frac{1}{\tau_{0}}\left(\beta_{0}(x)-\beta^{0}\right)=\beta_{1}(x) .
\end{aligned}
$$

We calculate $\vartheta$ from Eq. (1.7), using (1.1) and (2.1). Then

$$
\vartheta=\vartheta^{0} \exp \left\{\frac{\tau_{0}}{\vartheta^{0}}\left(-\beta_{t}-\frac{1}{\tau_{0}} \beta+\frac{1}{\tau_{0}} \beta^{0}\right)\right\}=\vartheta(\xi),
$$

where for convenience we introduce the variable $\xi$ defined by

$$
\xi=\beta_{t}+\frac{1}{\tau_{0}} \beta
$$

We express the coefficients in (1.6) in terms of $\xi$, so that

$$
\begin{aligned}
& C\left(\beta, \beta_{t}\right)=\tau_{0} g(\xi), \\
& H\left(\beta, \beta_{t}\right)=-g(\xi) \beta_{t},
\end{aligned}
$$

and

$$
g(\xi)=\rho_{0} \vartheta(\xi) c_{V}(\vartheta(\xi))>0
$$

Next we substitute these coefficients into Eq. (1.5) and multiply by $\tau_{0}^{-1}$, which gives

$$
-g(\xi) \beta_{t t}+k_{0} \beta_{x} \beta_{x t}+\frac{k_{0} \vartheta^{0}}{\tau_{0}} \beta_{x x}-\frac{1}{\tau_{0}} g(\xi) \beta_{t}=0
$$

If we define $P(\xi)$ such that

$$
P^{\prime}(\xi)=g(\xi)>0
$$

then

$$
P(\xi)_{t}=g(\xi) \xi_{t}
$$


On using (2.8), Eq. (2.6) can be written in the form

$$
\left(\frac{k_{0} \vartheta^{0}}{\tau_{0}} \beta_{x}\right)_{x}=\left(P(\xi)-\frac{1}{2} k_{0}\left(\beta_{x}\right)^{2}\right)_{t}
$$

Let $\phi(t, x)$ be a potential function such that

$$
\begin{aligned}
\phi_{x} & =P(\xi)-\frac{1}{2} k_{0}\left(\beta_{x}\right)^{2} \\
\phi_{t} & =\frac{k_{0} \vartheta^{0}}{\tau_{0}} \beta_{x} .
\end{aligned}
$$

Our next step is to obtain an equation for $\phi(t, x)$. First, on differentiating (2.10), we obtain

$$
\phi_{t t}-\frac{k_{0} \vartheta^{0}}{\tau_{0} g(\xi)} \phi_{x x}-\frac{\tau_{0}}{2 \vartheta^{0} g(\xi)}\left(\phi_{t}\right)_{x}^{2}+\frac{1}{\tau_{0}} \phi_{t}=0 .
$$

The variable $\xi$ can be expressed in terms of $\phi_{x}$ and $\phi_{t}$ from Eq. (2.10), namely,

$$
\xi=P^{-1}(\nu)
$$

where

$$
\nu=\phi_{x}+\frac{1}{2} \frac{\tau_{0}^{2}}{k_{0}\left(\vartheta^{0}\right)^{2}}\left(\phi_{t}\right)^{2}
$$

since the function $P$ is invertible (cf. (2.7)).

We define a function $\sigma(\nu)$, satisfying the relation

$$
\sigma^{\prime}(\nu)=\frac{k_{0} \vartheta^{0}}{\tau_{0} g\left(P^{-1}(\nu)\right)}>0 .
$$

As a result, we have the following second-order quasi-linear wave equation containing first-order dissipation,

$$
\phi_{t t}-\sigma(\nu)_{x}+\frac{1}{\tau_{0}} \phi_{t}=0
$$

with the initial conditions

$$
\phi(0, x)=\phi_{0}(x), \quad \phi_{t}(0, x)=\phi_{1}(x),
$$

where $\nu$ is given by $(2.13)$.

The initial data for $\phi(t, x)$ can be obtained from (2.2) via (2.10).

This equation is now in the form analyzed by Nishida [12]. Equation (2.15) will possess (cf. Nishida [12], Matsumara [11]) global smooth solutions $\phi(t, x) \in C^{2}\left(\mathbb{R}^{+} \times \mathbb{R}\right)$ for $\left|\phi_{0}(\cdot)\right|_{C_{2}}+\left|\phi_{1}(\cdot)\right|_{C^{1}}$ sufficiently small provided the function $\sigma$ satisfies the conditions (cf. $(2.14))$

$$
\begin{aligned}
\sigma(0) & =0, \\
\sigma^{\prime}(\nu) & \geq \sigma_{0}=\text { const. }>0, \quad \text { and } \sigma(\cdot) \in C^{4} .
\end{aligned}
$$

The solutions $\phi(t, x)$ have the property

$$
\|\phi(t)\|_{2} \equiv|\phi(t, \cdot)|_{C^{2}}+\left|\phi_{t}(t, \cdot)\right|_{C^{1}}+\left|\phi_{t t}(t, \cdot)\right|_{C^{0}}<\infty \quad \text { for all } t>0
$$


This result is obtained using energy estimates in spaces of $L^{2}$-functions together with Sobolev's Lemma in one space dimension.

We next show that a solution of Eq. (1.5) (equivalently (2.6) with (2.4)) satisfies, similarly,

$$
\|\beta(t)\|_{2} \equiv|\beta(t, \cdot)|_{C^{2}}+\left|\beta_{t}(t, \cdot)\right|_{C^{1}}+\left|\beta_{t t}(t, \cdot)\right|_{C^{0}}<\infty \quad \text { for all } t>0 .
$$

Using (2.17) and Eq. $(2.10)_{2}$, we have that $\beta_{x}, \beta_{x x}$, and $\beta_{x t}$ are bounded in $C^{0}$.

Now we will see that $\beta_{t}$ and $\beta_{t t}$ are also bounded, which will give (2.18), under the additional assumption

$$
P^{\prime}(\xi) \geq P_{0}=\text { const. }>0 .
$$

Equation $(2.10)_{1}$ implies $P(\xi)$ is bounded, which in turn implies that $\xi$ is bounded, under the assumption (2.19). Thus, there is a constant $\mathcal{D}$ for $t \in[0, T], T<\infty$, such that (cf. Definition (2.4))

$$
-\mathcal{D}<\beta_{t}+\frac{1}{\tau_{0}} \beta<\mathcal{D} .
$$

This implies the following inequality:

$$
-\mathcal{D} e^{\frac{t}{\tau_{0}}}<\left(\beta e^{\frac{t}{\tau_{0}}}\right)_{t}<\mathcal{D} e^{\frac{t}{\tau_{0}}}
$$

By integration of (2.21) we obtain upper and lower bounds for $\beta$, and by (2.20), for $\beta_{t}$.

Let us differentiate $(2.10)_{1}$ with respect to $t$ to give

$$
\phi_{x t}=P^{\prime}(\xi) \xi_{t}-k_{0} \beta_{x} \beta_{x t},
$$

which shows that in view of (2.19), and the boundedness of $\phi_{x t}, \beta_{x}$, and $\beta_{x t}, \xi_{t}=\beta_{t t}+$ $\tau_{0}^{-1} \beta_{t}$ is bounded. This together with our prior result gives the boundedness of $\beta_{t t}$.

Similar arguments can be applied to show that from the initial conditions, $\left|\beta_{0}(\cdot)\right|_{C^{2}}+$ $\left|\beta_{1}(\cdot)\right|_{C^{1}}$ is small if and only if $\left|\phi_{0}(\cdot)\right|_{C_{2}}+\left|\phi_{1}(\cdot)\right|_{C^{1}}$ is small. The above arguments imply the following:

Lemma. The Cauchy problem for Eq. (1.5) with (1.7) and (2.1) together with data (2.2) will have global smooth solutions $\beta(t, x) \in C^{2}\left(\mathbb{R}^{+} \times \mathbb{R}\right)$ for $\left|\beta_{0}(\cdot)\right|_{C^{2}}+\left|\beta_{1}(\cdot)\right|_{C^{1}}$ sufficiently small. Then

$$
\|\beta(t)\|_{2} \equiv|\beta(t, \cdot)|_{C^{2}}+\left|\beta_{t}(t, \cdot)\right|_{C^{1}}+\left|\beta_{t t}(t, \cdot)\right|_{C^{0}}<\infty \quad \text { for } t>0,
$$

provided that

$$
A_{0} \leq \vartheta(\xi) c_{V}(\vartheta(\xi)) \leq B_{0} \quad \text { and } c_{V}(\cdot) \in C^{3}
$$

where

$$
A_{0}=\text { const. }>0, \quad B_{0}=\text { const. }>0,
$$

and $\vartheta$ is given by (2.3) with (2.4).

The constants $A_{0}$ and $B_{0}$ are determined such that the assumptions $(2.16)_{2}$ and (2.19) are satisfied,

$$
\begin{aligned}
& A_{0}=P_{0} / \rho_{0}, \\
& B_{0}=k_{0} \vartheta^{0} /\left(\tau_{0} \sigma_{0} \rho_{0}\right) .
\end{aligned}
$$


3. Breakdown of smooth solutions. We will continue with the discussion of Eq. (2.6) under the assumption that the function $g(\xi)=c_{0}=$ const. Then the function $\sigma(\nu)$ defined by $(2.14)$ with condition $(2.16)_{1}$ is linear,

$$
\sigma(\nu)=a_{0} \nu
$$

and Eq. (2.15) takes the form

$$
\phi_{t t}-\left(a_{0} \phi_{x}+\frac{b_{0}}{2 a_{0}} \phi_{t}^{2}\right)_{x}+\frac{1}{\tau_{0}} \phi_{t}=0
$$

where the constants $a_{0}$ and $b_{0}$ are introduced for convenience,

$$
\begin{aligned}
a_{0} & =\frac{k_{0} \vartheta^{0}}{\tau_{0} c_{0}}, \\
b_{0} & =\frac{k_{0}}{c_{0}} .
\end{aligned}
$$

The corresponding system of equations for $w$ and $p$ (cf. (1.9)) can be reduced to $2 \times 2$ form

$$
\begin{gathered}
w_{t}-b_{0} p w_{x}-a_{0} p_{x}+\frac{1}{\tau_{0}} w=0, \\
p_{t}-w_{x}=0
\end{gathered}
$$

with initial data

$$
\begin{gathered}
w(x, 0)=w_{0}(x), \\
p(x, 0)=p_{0}(x) .
\end{gathered}
$$

The above system is of nonconservative form and includes dissipation. We will use Riemann invariants along characteristics to establish breakdown of solutions. This approach has been applied successfully to other problems in the literature; see Slemrod [13], MalekMadani and Nohel [10], Hattori [3], MacCamy [9], Longwei and Yongshu [8]. Another approach, used by Kosinski [5] for nonhomogeneous hyperbolic systems, cannot be applied because the system (3.4) does not satisfy the assumptions needed in [5] to show blow up.

The system (3.4) is strictly hyperbolic for any values of $p$ with eigenvalues:

$$
\begin{aligned}
& \lambda^{1}=\frac{1}{2}\left(-b_{0} p+\sqrt{b_{0}^{2} p^{2}+4 a_{0}}\right)>0, \\
& \lambda^{2}=\frac{1}{2}\left(-b_{0} p-\sqrt{b_{0}^{2} p^{2}+4 a_{0}}\right)<0,
\end{aligned}
$$

and characteristic curves, parametrized with $\gamma$ and $\delta$, respectively,

$$
\begin{aligned}
\frac{d}{d t} x_{1}(t, \gamma)=\lambda^{1}, & x_{1}(0, \gamma)=\gamma \\
\frac{d}{d t} x_{2}(t, \delta)=\lambda^{2}, & x_{2}(0, \delta)=\delta
\end{aligned}
$$


The Riemann invariants form a one-to-one transformation from $(w, p) \in \mathbb{R} \times \mathbb{R}$ to $(r, s) \in$ $\mathbb{R} \times \mathbb{R}:$

$$
\begin{aligned}
& r(w, p)=w+\int_{0}^{p} \lambda^{1}(z) d z \\
& s(w, p)=w+\int_{0}^{p} \lambda^{2}(z) d z .
\end{aligned}
$$

Next, we will use the following notation for the derivative along the characteristic $x_{1}$ or $x_{2}$ :

$$
\begin{aligned}
& \prime=\frac{\partial}{\partial t}+\lambda^{2} \frac{\partial}{\partial x} \\
& =\frac{\partial}{\partial t}+\lambda^{1} \frac{\partial}{\partial x}
\end{aligned}
$$

Then the Riemann invariants satisfy

$$
\begin{aligned}
r^{\prime} & =-\frac{1}{\tau_{0}} w \\
s^{\prime} & =-\frac{1}{\tau_{0}} w .
\end{aligned}
$$

We combine (3.8) and (3.6),

$$
\begin{aligned}
& r-s=\int_{0}^{p} \sqrt{b_{0}^{2} z^{2}+4 a_{0}} d z=F(p) \\
& r+s=2 w-\frac{b_{0}}{2} p^{2}
\end{aligned}
$$

which gives (3.10) written in terms of $r$ and $s$ :

$$
\begin{aligned}
& r^{\prime}=-\frac{1}{2 \tau_{0}}(r+s)-\frac{b_{0}}{4 \tau_{0}} p^{2}, \\
& s^{\prime}=-\frac{1}{2 \tau_{0}}(r+s)-\frac{b_{0}}{4 \tau_{0}} p^{2},
\end{aligned}
$$

where by Eq. $(3.11)_{2}, p=F^{-1}(r-s)$.

Next we will show that $r_{x}$ and $s_{x}$ develop singularities in finite time if $r_{0, x}$ and $s_{0, x}$ are sufficiently large, and so $w$ and $p$ cannot be in $C^{1}(\mathbb{R})^{2}$ for all time. The following arguments used are based on work by Lax [7] for nondissipative $2 \times 2$ systems, and for dissipative systems by Nishida [12], Slemrod [13], and others. One important difference here is that our eigenvalues do not satisfy the usual condition $\lambda^{1}=-\lambda^{2}$; so in Eq. (3.12) we have an extra nonlinear term.

LEmmA. Let $\left|r_{0}\right|=\sup _{x \in \mathbb{R}}\left|r_{0}(x)\right|,\left|s_{0}\right|=\sup _{x \in \mathbb{R}}\left|s_{0}(x)\right|$. Then, as long as smooth solutions exist, we have

$$
\sup _{x \in \mathbb{R}}|r(x, t)|+\sup _{x \in \mathbb{R}}|s(x, t)| \leq\left(\left|r_{0}\right|+\left|s_{0}\right|\right) e^{\frac{2}{\tau_{0}} t}
$$


Proof. We rewrite the function $F(p)=r-s$ in Eq. $(3.11)_{1}$ in more convenient variables $\zeta$ and $z$ defined by

$$
\zeta=\frac{b_{0}}{2 a_{0}}(r-s), \quad z=\frac{b_{0}}{\sqrt{4 a_{0}}} p .
$$

Then

$$
\zeta=\mathcal{F}(z)=z \sqrt{z^{2}+1}+\log \left(z+\sqrt{z^{2}+1}\right) .
$$

Straightforward calculations lead to the observation

$$
|z| \leq \sqrt{|\zeta|}
$$

and by (3.13),

$$
|p| \leq \sqrt{\frac{2}{b_{0}}|r-s|} .
$$

Now we integrate both equations in (3.12) along their corresponding characteristics,

$$
\begin{aligned}
& r\left(x_{2}(t, \delta), t\right)=r_{0}(\delta)-\frac{1}{2 \tau_{0}} \int_{0}^{t}\left\{r\left(x_{2}(\tau, \delta), \tau\right)+s\left(x_{2}(\tau, \delta), \tau\right)-\frac{b_{0}}{2} p^{2}\left(x_{2}(\tau, \delta), \tau\right)\right\} d \tau, \\
& s\left(x_{1}(t, \gamma), t\right)=s_{0}(\gamma)-\frac{1}{2 \tau_{0}} \int_{0}^{t}\left\{r\left(x_{1}(\tau, \gamma), \tau\right)+s\left(x_{1}(\tau, \gamma), \tau\right)-\frac{b_{0}}{2} p^{2}\left(x_{1}(\tau, \gamma), \tau\right)\right\} d \tau,
\end{aligned}
$$

where, by Eq. (3.7), characteristic curves are given by

$$
\begin{aligned}
& x_{2}(t, \delta)=\delta+\int_{0}^{t} \lambda^{2} d \tau, \\
& x_{1}(t, \gamma)=\gamma+\int_{0}^{t} \lambda^{1} d \tau .
\end{aligned}
$$

Equations (3.16) and (3.15) imply the inequalities

$$
\begin{aligned}
& \left|r\left(x_{2}(t, \delta), t\right)\right| \leq\left|r_{0}(\delta)\right|+\frac{1}{\tau_{0}} \int_{0}^{t}\left\{\left|r\left(x_{2}(\tau, \delta), \tau\right)\right|+\left|s\left(x_{2}(\tau, \delta), \tau\right)\right|\right\} d \tau \\
& \left|s\left(x_{1}(t, \gamma), t\right)\right| \leq\left|s_{0}(\gamma)\right|+\frac{1}{\tau_{0}} \int_{0}^{t}\left\{\left|r\left(x_{1}(\tau, \gamma), \tau\right)\right|+\left|s\left(x_{1}(\tau, \gamma), \tau\right)\right|\right\} d \tau .
\end{aligned}
$$

We define

$$
\begin{aligned}
R(t) & =\sup _{x \in \mathbb{R}}|r(x, t)|, \\
S(t) & =\sup _{x \in \mathbb{R}}|s(x, t)| .
\end{aligned}
$$

Then it follows from (3.18) that

$$
\begin{aligned}
& R(t) \leq\left|r_{0}\right|+\frac{1}{\tau_{0}} \int_{0}^{t}\{R(\tau)+S(\tau)\} d \tau, \\
& S(t) \leq\left|s_{0}\right|+\frac{1}{\tau_{0}} \int_{0}^{t}\{R(\tau)+S(\tau)\} d \tau .
\end{aligned}
$$


Then

$$
R(t)+S(t) \leq\left|r_{0}\right|+\left|s_{0}\right|+\frac{2}{\tau_{0}} \int_{0}^{t}\{R(\tau)+S(\tau)\} d \tau,
$$

and finally, by Gronwall's inequality,

$$
R(t)+S(t) \leq\left(\left|r_{0}\right|+\left|s_{0}\right|\right) e^{\frac{2}{\tau_{0}} t}
$$

which establishes the lemma.

THEOREM. If $r_{0, x}$ or $s_{0, x}$ are sufficiently large for some $x \in \mathbb{R}$, then any $C^{1}(\mathbb{R})^{2}$ solution $(w, p)$ to system (3.4) only exists for finite time.

Proof. The functions $\lambda^{1}(p)$ and $\lambda^{2}(p)$ can be expressed as functions of $(r-s)$ by (3.11). Thus $\lambda^{1}=\lambda^{1}(r-s), \lambda^{2}=\lambda^{2}(r-s)$. Differentiating Eqs. (3.10) with respect to $x$ implies

$$
\begin{gathered}
r_{x}^{\prime}+\lambda_{r}^{2} r_{x}^{2}+\lambda_{s}^{2} r_{x} s_{x}+\frac{1}{\tau_{0}} w_{x}=0 \\
s_{x}{ }^{`}+\lambda_{s}^{1} s_{x}^{2}+\lambda_{r}^{1} r_{x} s_{x}+\frac{1}{\tau_{0}} w_{x}=0 .
\end{gathered}
$$

Now we use the relations

$$
r_{x}=\frac{1}{\lambda^{1}-\lambda^{2}}\left(r^{`}-s^{`}\right), \quad s_{x}=\frac{1}{\lambda^{1}-\lambda^{2}}\left(r^{\prime}-s^{\prime}\right)
$$

together with

$$
w_{x}=\frac{\lambda^{1}}{\lambda^{1}-\lambda^{2}} s_{x}-\frac{\lambda^{2}}{\lambda^{1}-\lambda^{2}} r_{x}
$$

We will proceed with the proof based on Eq. $(3.20)_{1}$, concentrating on the value of $r_{x}$. The argument applied here could be applied in a similar way to Eq. $(3.20)_{2}$ for $s_{x}$.

Multiplication of both sides of $(3.20)_{1}$ by the integrating factor $\mu=\mu(r-s)$ defined by

$$
\mu(r-s)=\exp \left(-\int_{0}^{r-s}\left(\frac{\lambda_{r}^{2}}{\lambda^{1}-\lambda^{2}}\right)(\zeta) d \zeta\right)
$$

yields

$$
\left(\mu r_{x}\right)^{\prime}+\lambda_{r}^{2} \mu r_{x}^{2}-\frac{\lambda^{2}}{\tau_{0}\left(\lambda^{1}-\lambda^{2}\right)} \mu r_{x}+\frac{\lambda^{1}}{\tau_{0}\left(\lambda^{1}-\lambda^{2}\right)^{2}} \mu\left(r^{\prime}-s^{\prime}\right)=0 .
$$

We now follow Slemrod [13]. Let us define

$$
f(r-s)=-\int_{0}^{r-s}\left(\frac{\lambda^{1}}{\left(\lambda^{1}-\lambda^{2}\right)^{2}} \mu\right)(\zeta) d \zeta
$$

and

$$
\theta=\mu r_{x}
$$

so that Eq. (3.24) becomes

$$
\theta^{\prime}=-\lambda_{r}^{2} \mu^{-1} \theta^{2}+\frac{\lambda^{2}}{\tau_{0}\left(\lambda^{1}-\lambda^{2}\right)} \theta+\frac{1}{\tau_{0}} f^{\prime}
$$


Setting

gives us

$$
\begin{gathered}
\hat{\theta}=\theta-\frac{1}{\tau_{0}} f, \\
k_{1}(r-s)=-\lambda_{r}^{2} \mu^{-1}>0, \\
k_{2}(r-s)=-\frac{\lambda^{2}}{\lambda^{1}-\lambda^{2}}>0
\end{gathered}
$$

Define

$$
\hat{\theta}^{\prime}=k_{1} \hat{\theta}^{2}+\frac{1}{\tau_{0}} k_{2}\left[2 \frac{k_{1}}{k_{2}} f-1\right] \hat{\theta}+\frac{k_{1}}{\tau_{0}^{2}} f^{2}-\frac{k_{2}}{\tau_{0}^{2}} f
$$

$$
\begin{gathered}
\varepsilon=\inf k_{1} \geq 0, \quad M=\sup \left|\frac{1}{\tau_{0}} k_{2}\left[2 \frac{k_{1}}{k_{2}} f-1\right]\right|, \\
K=\inf \left(-\frac{k_{2}}{\tau_{0}^{2}} f\right) .
\end{gathered}
$$

Then

$$
\frac{1}{\tau_{0}} k_{2}\left[2 \frac{k_{1}}{k_{2}} f-1\right] \hat{\theta} \geq-\frac{1}{2}\left(\varepsilon \hat{\theta}+\frac{M^{2}}{\varepsilon}\right)
$$

and

$$
k_{1} \hat{\theta}^{2}+\frac{1}{\tau_{0}} k_{2}\left[2 \frac{k_{1}}{k_{2}} f-1\right] \hat{\theta}+\frac{k_{1}}{\tau_{0}^{2}} f^{2}-\frac{k_{2}}{\tau_{0}^{2}} f \geq \frac{\varepsilon}{2} \hat{\theta}^{2}-\frac{M}{2 \varepsilon}+K .
$$

Without loss of generality, suppose $K \leq 0$.

Next we compare two solutions $\hat{\theta}$ of Eq. (3.29) and $\tilde{\theta}$ for which $\hat{\theta}(x, 0)=\tilde{\theta}(x, 0)=\theta_{0}$, where

$$
\frac{d}{d t} \tilde{\theta}=\frac{\varepsilon}{2} \tilde{\theta}^{2}-\frac{M}{2 \varepsilon}+K
$$

and for which $\hat{\theta}(t) \geq \tilde{\theta}(t)$ for some $t \in[0, d)$.

The solution $\tilde{\theta}(t)$ of Eq. (3.33) is given by

$$
\tilde{\theta}(t)=\sqrt{\frac{A}{B}} \frac{1+D_{0} e^{2 \sqrt{A B} t}}{1-D_{0} e^{2 \sqrt{A B} t}}
$$

where

$$
\begin{gathered}
A=\frac{\varepsilon}{2}, \quad B=\frac{M}{2 \varepsilon}-K, \quad D_{0}=\frac{\theta_{0}-\sqrt{\frac{A}{B}}}{\theta_{0}+\sqrt{\frac{A}{B}}}, \\
\theta_{0}=\mu\left(r_{0}-s_{0}\right) r_{0, x}-\frac{1}{\tau_{0}} f\left(r_{0}-s_{0}\right) .
\end{gathered}
$$

From this, it follows that $\tilde{\theta}\left(x_{2}(t, \delta), t\right)$, and so $\hat{\theta}\left(x_{2}(t, \delta), t\right)$, tends to infinity in finite time $t^{*}$ if $0<D_{0}<1$ or, equivalently,

$$
0<\frac{2 G}{\mu\left(r_{0}-s_{0}\right) r_{0, x}-\frac{1}{\tau_{0}} f\left(r_{0}-s_{0}\right)+G}<1
$$

where $G=\sqrt{A / B}$.

Equation (3.36) is satisfied for $r_{0, x}$ sufficiently large. Since $\mu(r-s) r_{x}=\hat{\theta}+\frac{1}{\tau_{0}} f$, thus $\mu(r-s) r_{x}$ goes to infinity. From the lemma, $\mu(r-s)$ is bounded for $t \leq t^{*}$, and so $r_{x}$ goes to infinity. 


\section{REFERENCES}

[1] V. A. Cimmelli and W. Kosinski, Heat waves in continuous media at low temperatures, Quaderni del Dipartimento di Matematica, vol. 6, Universaita della Basilicata, Potenza, 1992

[2] V. A. Cimmelli and W. Kosinski, Well posedness for a nonlinear hyperbolic heat equation, Ricerche di Matematica 42, 49-68 (1993)

[3] H. Hattori, Breakdown of smooth solutions in dissipative nonlinear hyperbolic equations, Quart. Appl. Math. 40, 113-127 (1982)

[4] W. Kosinski, Elastic waves in the presence of a new temperature scale, Elastic wave propagation, M. F. McCarthy and M. A. Hayes, eds., Elsevier Science, North-Holland, Amsterdam, 1989, p. 629

[5] W. Kosinski, Gradient catastrophe in the solution of nonconservative hyperbolic systems, J. Math. Anal. Appl. 61, 672-688 (1977)

[6] W. Kosinski and K. Saxton, The effect on finite time breakdown due to modified Fourier laws, Quart Appl. Math. 51, 55-68 (1993)

[7] P. D. Lax, Development of singularities of solutions of nonlinear hyperbolic partial differential equations, J. Math. Phys. 5, 611-613 (1964)

[8] L. Longwei and Z. Yongshu, Existence and non-existence of global smooth solutions for quasilinear hyperbolic systems, Chinese Ann. of Math. 9B, 372-377 (1988)

[9] R. C. MacCamy, An integro-differential equation with application in heat flow, Quart. Appl. Math. 35, 1-19 (1977)

[10] R. Malek-Madani and J. A. Nohel, Formation of singularities for a conservation law with memory, Siam J. Math. 16, 530-540 (1985)

[11] A. Matsumara, Global existence and asymptotic solutions of the second order quasi-linear hyperbolic equations with first order dissipation, Publ. Res. Inst. Math. Sci. Kyoto Univ. A 13, 349-379 (1977)

[12] T. Nishida, Nonlinear hyperbolic equations and related topics in fluid dynamics, Publications Mathématiques D'Orsay, Université de Paris-Sud, Département de Mathématiques, vol. 78.02, 1978

[13] M. Slemrod, Instability of steady shearing flow in a nonlinear viscoelastic fluid, Arch. Rational Mech. Anal. 68, 211-225 (1978) 